\title{
Las elecciones en el Cádiz de 1881: el comienzo del turno liberal
}

\author{
JOSE MARCHENA DOMNGUEZ
}

En nuestra comunicación, intentamos ofrecer un avance de una investigación más exhaustiva que actualmente desarrollamos, sobre el tema electoral y caciquil en el Cádiz de la Restauración. En concreto, desglosamos los aspectos más representativos de la diná mica electoral acaecida en la ciudad andaluza durante 1881. Año este que marca el principio del llamado "Turno de Partidos", y en el qu empiezan a desarrollarse la impronta característica que marcará prácticamente todo el periodo político aludido. Nuestro esquema de desarrollo, escueto en la medida de la corta extensión de rigor, establecerá en primer lugar una introducción del momento político al que llega la ciudad gaditana en este año de 1881,para seguir por un análisis de los comicios electorales, en sus vertientes municipales, provinciales y nacionales; apreciaciones en torno al discurso electoral y político desarrollado en los respectivos órganos de prensa gaditano.

La llegada del nuevo periodo político conocido como la Restauración borbónica, había ssorprendido• a la ciudad gaditana con una situación de clarísima precariedad socioeconómica. Al desmantelamiento portuario y comercial se le sumaba la falta de alternativa en el ramo secundario -ausencia de industrias y factorías agravadas por la llamada ezona polémican- y la de un término municipal que compensara con un potencial agro, la penuria y crisis galopante. De esta manera y ya desde el inicio del funcionamiento en 1876 de los gabinetes representativos, los respectivos políticos intentan denodadamente aliviar las innumerables necesidades de la ciudad y población. De esta manera, y durante el periodo comprendido entre 1876 a 1881 , vemos asomar en los gabinetes gaditanos de representación, a una serie de personajes y cabezas visibles de innegable pasado en formaciones de corte moderado y monárquico, pero más importante aún, los jóvenes valores que cubrirán la primera etapa del periodo de la Restauración, en lo referente a los representantes del partido conservador canovista. Hombres que llegan a cobrar auténti- 
co olor de notables y patricios locales. Hombres pertenecientes a estirpes burguesas de arraigo en Cádiz. ${ }^{1}$

El año de 1881, era el año en el que, con la entrada del Partido Liberal en el gobierno, se iniciaba la galvanización del sistema de turnos promovido por Cánovas del Castillo. De tal guisa, los resortes electorales, en sus variantes municipales, provinciales y nacionales, no variaron en gran cosa respecto a los primeros comicios de la Restauración. ${ }^{2}$

En el espectro administrativo gaditano el ejercicio de 1881 se inicia con una serie de acciones que determinan en cierta medida el comienzo de un año electoral muy movido. Así, haciendo acopio de la potestad designativa del Gobierno Central y sus órganos provinciales -Gobiernos Civiles-, el titular de Cádiz Arderius suspendía entre otros cargos al alcalde de la ciudad el conservador José Morales Borrero a diversos diputados provinciales por causas poco claras y a aquellos vocales de la Comisión Provincial -órgano representativos que entre otras competencias ostentaban la de nombrar concejales interinos en los tercios vacantes-, que podían suponer obstáculos a los intereses liberales -también conservadores como Regife, Ferrer, Mier o Pongilioni. Todo ello, ante la indignación de la prensa y los órganos de afines. ${ }^{3}$

Decía al respecto el rotativo conservador El Comercio:

Aquí se ganan las elecciones por medios oficiales cuando de ese modo se desafía a la opinión pública, pretendiendo imponerla un yugo que rechaza la noble y digna altivez del cuerpo electoral. Siga el señor Arderius por ese camino, siga acomodando sus actos a las exigencias de las personas que le rodean, siga organizando los pueblos, a gusto de los

(1) Son los casos entre otros del valenciano afincado en Cádiz Eduardo J. Genovés, primer presidente de la Diputación de Cádiz en este periodo, del bienhechor burgués José Moreno de Mora, del poeta y periodista Arístides Pongilioni, de José Huidobro, de José Ramón de Santa Cruz, de José de la Viesca primer Alcalde de la Restauración, del tratadista y economista gaditano José Luis Díez, de Enrique Díaz Rocafull, de José Morales Borrero, del polifacético Salvador Viniegra, de Antonio RuizTagle,etcétera.V.Actas Capitulares del Ayuntamiento de Cádiz, Actas provinciales y Diario de Sesiones, para corroborar las formaciones representativas de la ciudad.

(2) En realidad, a nivel nacional las elecciones sólo variaron en cuanto a los protagonistas, que en este caso fueron el Presidente Sagasta y su Ministro de Gobernación Venancio González. En cualquier caso, la Ley del 2 de octubre de 1877, aseguraba un total control del gobierno central en las elecciones y composiciones de las distintas corporaciones. V. MARTINEZ MARIN, A. La Representatividad municipal española .Murcia, Universidad, 1989, y GONZALEZ MUÑIZ, M.A.Constituciones, Cortes y Elecciones españolas. Madrid, Júcar, 1978.

(3) La suspensión total o parcial de Ayuntamientos previa a las elecciones del mismo año no fue un fenómeno aislado en Cádiz y su Provincia. Se habla de medio millar de corporaciones alteradas en toda España, al amparo de la disposición legal, por la que un tercio de las corporaciones podian ser consideradas vacantes seis meses antes de las elecciones. Ibidem y El Comercio, 17 y 24-mar.-y 2-ab.-1881. 
mandarines del día, y ya verá el fruto que recoge en favor del gobierno [...] gobernadores de esa fuerza son un hallazgo inapreciable para todo un partido de oposición."

Efectivamente, el Partido Conservador; consciente de la hegemonía liberal que ahora se avecinaba, descubría en la persona del nuevo alcalde interino Antonio Alvarez Jiménez, el exponente de las nuevas necesidades del partido en el poder, al margen de

-...las tareas de la administración local, que no piensa en mejoras ni adelantos de ningún género.... No obstante, no se desanimaban a seguir en la contienda electoral, máxime con las fuerzas que presumiblemente iban a dar a grupo liberal, muchos quebraderos de cabeza. ${ }^{4}$

A finales del mes de abril se iniciaba la contienda electoral municipal con un clima de ataques mútuos entre los órganos de los dos grandes partidos del sistema. Si bien en el caso de los conservadores las críticas se centraban en denunciar el menor resquicio de irregularidad se remarcaba la iniciativa del Alcalde interino de anular las cédulas antiguas como vía de control y manipulación del censo-, los liberales a través de su órgano El Eco de Cádiz, manifestaban sentirse dispuestos a ganar unas elecciones limpiamente, a través del voto libre y espontáneo del electorado, y no con los amaños y abusos que desde los últimos seis años utilizaban los seguidores de Cánovas del Castillo.

Con tales ingredientes, ambos partidos publicaban a primeros de mayo sus candidatos para el consistorio gaditano. Por parte liberal, las lista de candidatos estaba integrada por una mezcla de nuevos valores para el futuro y algunos políticos participantes en las etapas isabelina y del sexenio revolucionario, como Palacios o Herrero:

$\begin{array}{ll}\text { Manuel A. Amusátegui } & \text { José } M^{2} \text { Rivas Morenati } \\ \text { Nicomedes Herrero y Cuesta } & \text { José Palacios y Muro } \\ \text { José Ruiz y Ruiz } & \text { Jerónimo Girau } \\ \text { Jorge Ridruejo y Pueyo } & \text { Francisco Martínez } \\ \text { Enrique del Toro } & \text { Miguel Aguado y Cruz } \\ \text { José Calatrigo y Verdugo } & \text { Rafael Mato Berman } \\ \text { José M } M^{3} \text { Requejo Gastardi } & \text { Fco.de la Peña Catalán } \\ \text { Miguel Portillo Palomo } & \text { Luis Morón y Alvarez } \\ \text { Fco. Dolareo y Roguez. Sánchez } & \text { José Díaz y González } \\ \text { Dionisio Ruiz Alcázar. } & \end{array}$

(4) El Partido Conservador gaditano hacía frente a los ataques de los liberales de haber desarrollado anteriores mandatos, al amparo de elecciones amañadas. Sobre los calificativos de insulto ironizaba: ; ellos tan liberales, tan expansivos, tan tolerantes, como se está viendo en nuestra provincia y en todos los de España! ; Nosotros tan reaccionarios, tan exclusivos, tan intransigentes como lo atestiguan los mismos constitucionales..... El Comercio, 9-ab.-1881. 
En cuanto a los candidatos conservadores, se presenta una lista circunstancial con muy pocos elementos de viejas corporaciones desde 1876 - tan sólo los casos de Ruiz-Tagle, Soulé, Genovés, de la Vega y Fernández Quarteroni-, y una mayoría de nuevos nombres en la órbita conservadora gaditana:

Carlos Perea y Orive

Manuel Natera Fernández

José $M^{a}$ Muñoz

Antonio López Martínez

Agustín García Gutiérrez

Antonio Ruiz-Tagle

Pedro Ibáñez Pacheco

Luis Chorro

Francisco $M^{\mathbf{a}}$ de la Vega

Benito García Quintana

Nicolás Fernández Quarteroni

Fernando García Arboleya

Miguel Moreno Martínez.

Ricardo González-Abreu

Eduardo J. Genovés

Francisco Meléndez

José $\mathrm{M}^{\mathrm{a}}$ Soulé

Santiago de la Torre. 5

Ricardo Girón Severini

Al comienzo electoral, le siguieron las correspondientes y airadas protestas de los conservadores. En particular, en las iniciales para determinar la composición de Mesas, donde los propios presidentes liberales expulsaron en Cádiz a diversos representantes conservadores, según éstos ....para que no pudiéramos ser testigos de sus fechorías, para quedarse solos y hacer en familia la elección. Señalaban no haber sido aceptada ninguna protesta a un Notario sin autoridad, e incluso el envío a prenvención del anterior Alcalde Morales Borrero. Se insta a la vía judicial, como vía rápida y eficaz ante una sucesión de irregularidades y acontecimientos violentos, que inundan otros distritos electorales de la provincia:

"No más impunidad para ciertos hechos que tienen en la ley su correspondiente sanción penal. Que no se rían por más tiempo de sus farsas los monopolizadores del sufragio público. A los tribunales con ellos y repare de una vez y para siempre si son o no son posibles las elercicnes en Cádiz cuando ocupan el poder los mandarines del día."

Aún en un tono de sorna, se maravillaba El Comercio, de la ppopularidad" de los fusionistas en zonas de la ciudad donde algunos como el caso de José Calatrigo llegan a sacar casi el $100 \%$ de los votos posibles, incluyendo a enfermos, "lázaros" o inexistentes:

*Asombra la popularidad del Partido Liberal Fusionista en Cádiz. En el barrio de Hércules hay 197 electores. Rebajando los muertos, ausentes,

(5) Otras formaciones fuera del Turno de Partidos como los Posibilistas de Castelar, también presentaron candidatos de reconocido bagaje en la historia de la democracia y el republicanismo gaditano, como los Federico Rudolph, José Arizmendi, Emilio Rodríguez García, José Jiménez-Mena y Alfonso Moreno Espinosa.C.El Eco de Cádiz, 13 y 21-ab.1881 y El Comercio, 1 y 2-may.-1881. 
desconocidos y materialmente imposibilitados de salir de sus casas, quedan en disposición de poder votar 152.

El candidato fusionista ha obtenido 151 votos. El voto restante se lo distribuyen como pueden los demás partidos políticos de la localidad. Hágase la misma cuenta en los otros barrios y resultará lo propio.

iQue popularidad la del partido fusionista!

iY nosotros hablamos de farsas!.. ${ }^{6}$

Por su turno, los fusionistas sabedores de llevar ahora el mango de la sartén política, arguyen entre otra razones a la derrota conservadora, como de haber ejercido una política raberrante", poco reformista y cerrada. Completan tal visión en la silueta de un partido que lejos de representar la opinión pública, sólo se dedicó a malgastar los medios de un poder detentado durante más de un lustro:

-...cuando la opinión pública se incluía en favor de estas o aquellas ideas, los hombres que las profesan pueden tener la completa seguridad de ver realizado su ideal; y si ya están en el poder y por consiguiente cuentan con los medios necesarios para ello, entonces pueden ver en tal inclinación la prueba más clara de que representan las ideas dominantes en la nación y de que sus actos como gobierno están en armonía con los que deben a su patria y a sus conciudadanos [...] además el Partido Liberal Conservador ha contado antes de las elecciones con la libertad suficiente para reunir y preparar sus huestes [...] y si se atribuyen [la derrotal a presiones y obstáculos ilegales debemos confesar, que dicho partido no representa la opinión pública, pues contra ésta, las coacciones y obstáculos son delgados hilos que facilmente se rompen."

De cualquier forma y como el sistema tenía previsto, el triunfo del Partido Liberal estaba cantado logrando sentar sus dieciocho representantes en el Consistorio gaditano, a los que se sumaban tres concejales de la formación posibilista: Arizmendi, Rodríguez y Rudolph. Los restantes candidatos conservadores lograron cifras exíguas -ninguno alcanzó la decena de votos-, por lo que el "pucherazo" fue totalmente redondo.

El 25 de junio de 1881, el gobierno liberal de Sagasta disolvía las Cortes para convocar elecciones el 21 de agosto para diputados, y el 2 de

(6) Merecen ser citadas las irregularidades habidas en otras zonas de la provincia como en el Puerto de Santa Maria, donde llegan las fuerzas públicas en estado ebrio a pegar y obligar a los votantes a ejercer su ederecho., siendo algunos de estos miembros del -orden., provenientes del presidio. En San Fernando llegan a retirarse conservadores y demócratas, debido a la gran cantidad de intrusos en la composición de las Mesas.

En realidad de poco servía la vía judicial cuando en algunos casos y no pocos, existían jueces adictos que garantizaban la consumación del manejo electoral, y por lo tanto la constitución de Mesas, votaciones, cédulas de votación y censos al gusto del -consumidor., estaban más que garantizadas. V. VARELA ORTEGA, J. Los Amigos Políticos.Madrid, Alianza, 1977,p.406,y El Comercio,2,3 y 6-may.-1881. 
septiembre para senadores. Nuevamente la máquina electoral se ponía en marcha, y nuevamente gozaban la vez las formaciones liberales.

En Cádiz y por segunda vez, estos comicios servían de pretexto a los conservadores gaditanos para advertir la manipulación efectuada por el partido de Sagasta. Durante toda la campaña electoral, el rotativo El Comercio, publicaba un pequeño anuncio en donde se describían como los seguidores liberales e incluso empleados municipales, arrancaban por las casas dobles firmas a los electores conservadores, en los pliegos para candidatos a Mesas, con lo que legalmente quedaban anuladas. Otras variantes de chantaje electoral también eran descritas: de nuevo representantes municipales, incluido alcaldes de barrio, sacaban por la fuerza las firmas de comerciantes con establecimientos comestibles, bajo la amenaza de inspecciones fiscales y sanitarias:

-A un elector de esta ciudad que tiene un establecimiento de bebidas, se le ha presentado el alcalde de barrio en que vive,exigiéndole su firma para interventor de la futura mesa electoral, en favor del Partido Liberal Fusionista. El elector a quien aludimos, en uso de su derecho, se negó a firmar, y a poco fue el mismo alcalde de barrio acompañado de un médico, en clase de perito para reconocer los vinos y licores de su establecimiento, con el pretexto de examinar su estado sanitario [...] esta es la libertad que prometen al cuerpo electoral los mandarines del día.*

Por su parte, y ante los insistentes rumores de la prensa fusionista de la retirada del grupo conservador, estos últimos desmentían afirmando que r...El Progreso se equivoca y que el Partido Conservador ha de dar mucho que hacer y que pensar a los amigos del colegan. Prometen llevar a cabo una estrategia reivindicativa y de protesta, no tan pasiva como las elecciones anteriores. Ante la hipotética inoperancia de la Comisión Provincial de Cádiz -que como ya anotamos fue adaptada al gusto liberal-, estarían dispuestos a llevar las posibles denuncias al Tribunal de Actas Graves del Congreso. ${ }^{7}$

En cuanto a los candidatos, desde un primer momento observamos como surgen dos tendencias dentro de las propuestas efectuadas por el Partido Liberal:la del Comité Local que defiende entre otros al General de Marina Florencio Montojo, o Carlos Rodríguez Batista, y la del Comité Provincial con nombres como José González de la Vega o Galindo. En realidad detrás de todos estos pronósticos,se encontraban las apetencias de dos de los principales cabezas visibles del fusionismo en la provincia. Por un lado González de la Vega y por otro un joven Cayetano del Toro,

(7) Cfr.El Clamor de Cádiz,6-may.-1881 y El Comercio, 22 y 23-jun.-,2, 27, 30 y 31-jul.-y 1 y 3-ag.-1881. 
al que ya la prensa de oposición calificaba de gran lumbrera del fusionismo gaditano.

Así, de la Vega se oponía a los candidatos de la otra fracción como Sánchez Mira y Moreno Rodríguez por la circunscripcción de Jerez y a Rodríguez Batista por la de Cádiz. De cualquier forma, la superioridad y mejor relación del gran oftalmólogo con los resortes centrales de Gobernación -era incluso gran amigo personal de Sagasta-, no dejaban duda de quien se impondría.

De nuevo la visión conservadora nos ofrece datos al respecto. Los innumerables rumores de candidatos sin confirmar empujan a romper una lanza en favor de los ignorados electores. *...lo más chistoso es que aquí no se cuenta para nada con los electores. Esto se comprende bien. ¿que falta hacen los electores? ¿no es mucho mejor vaciar el saco?. De tal forma, y a dos días de los comicios, el Partido Liberal no se había decidido aún por la lista definitiva, lo que reforzaba en opinión de El Comercio, el desinterés hacia un electorado que poco iba a ventilar en la contienda:

-Tampoco ayer publica El Progreso, la candidatura ministerial para la circunscripción de Cádiz. ¿A cuando se aguarda?.¿Es que ni el gobierno ni el comité tienen aquí candidatos? ¿O es que huye de la publicidad y no se cuenta para nada con la opinión?., ${ }^{8}$

Respecto a los candidatos conservadores -por Cádiz sólo presentan a Genovés-, su escaso eco y propaganda confirman la existencia de un sistema perfectamente cerrado. Quienes sí parten una original alternativa, serán los miembros de la Liga de Contribuyentes de Cádiz que, cansados de la inoperancia del sistema y de sus representantes, intentan presentar y llevar un candidato a Cortes en la persona de Bernardo Manuel de la Calle, con el fin de intentar subsanar los graves problemas de la ciudad gaditana y su Traspaís. Más originales aún si cabe, en la medida de presentar un auténtico programa electoral con programas publicados en el Clamor de Cádiz y con diversas reuniones públicas. Modalidad prácticamente no integrada en la maquinaria electoral de los partidos del Turno durante la Restauración.

Así, se toma como base el abrigar ....la esperanza que esta noble ciudad tendría por fin lo que hasta aquí no había tenido, esto es procuradores verdaderamente amantes de sus intereses». Ello venía precedido de

(8) El organigrama caciquil en lo referente a la dependencia de los organismos centrales del poder y a la diversidad de fracciones locales, queda reflejado en este modelo liberal gaditano. En concreto surge un interés por parte del Ministerio de Gobernación en situar en el distrito de El Puerto de Santa Maria al Conde de Niebla, si bien el otro candidato y futuro diputado Pedro Manjón logra ganarse el apoyo del titular de Fomento, bajo la excusa de *compromisos locales..Cfr.El Comercio, 3, 5, 20 y 21-ag.-1881. 
un programa muy acorde con las necesidades del momento de la ciudad. Necesidades que a pesar de los afanes administrativos de anteriores gabinetes, no habían llegado a soluciones reales. Así, puntos como la rectificación del censo tributario a la realidad socioeconómica de la ciudad, inicio de las Obras del Puerto, establecimientos de depósitos flotantes de carbón, edificación de viviendas e industrias en zonas de jurisdicción militar, impulso a las industrias salineras y marítimas. No olvidaba tampoco de exaltar en la medida de lo posible la figura de su candidato, a la vez que criticar el actual sistema electoral que no conduce sino a la indiferencia electoral. ${ }^{9}$

Con estos elementos, las elecciones transcurrieron en el clima típico de reclamaciones y chanchullos, arrojando los siguientes resultado:

Carlos Rodríguez Batista
Eduardo J. Genovés
José González de la Vega
Gral. Florentino Montojo
Juan Galindo
Bernardo María de la Calle

$\begin{array}{rc}971-978 & \text { votos } \\ 946-1140 & * \\ -835 & * \\ 544-545 & : \\ -340 & * \\ 18-50 & \times\end{array}$

En el resto de los distritos de la provincia, los triunfos fueron para los liberales Gutiérrez Agüera, Sánchez Mira, Alvarez Jiménez, Manjón y Ruiz Martínez, llamando la atención los inesperados triunfos del conservador Genovés en Cádiz y del posibilista Moreno Rodríguez en Jerez, al que lo conservadores lo achacaban a un entendemiento con los liberales, para evitar el triunfo de los canovistas Garrido y Camacho.

En cuanto al análisis de los resultados, el órgano conservador El Comercio, se sorprendía de la inverosimil remontada electoral del jefe liberal y presidente interino de la Diputación González de la Vega, donde de cuatro votos obtenidos en Cádiz, lograra quinientos de los quinientos catorce posibles en San Fernando, o bien como la Crónica, periódico liberal achacaba a un Génovés que obtenía más votos que nadie en la capital, desbancando al liberal Galindo.

(9) La Liga de Contribuyentes de Cádiz, fue una entidad que siendo de carácter privado, estuvo siempre velando por los intereses de los sectores ciudadanos implicados en el nivel económico, lo que en suma venía a ser una lucha por los intereses generales de la ciudad. Esta iniciativa se entiende dentro de la dinámica de una Liga que nunca vaciló en los momentos puntuales, de luchar y presionar a los gobiernos y órganos representativos, cuando estimaban una situación límite en la economía gaditana, cosa que para su desgracia sucedía a menudo. V.El Clamor de Cádiz,6 y 26-jul.-y 7, 19 y 20-ag.-1881.

(10) La primera cifra hace alusión a fuentes liberales y la segunda a las conservadoras.V.El Clamor de Cádiz, 22-ag.-1881 y El Comercio, 23-ag.-1881. 
De cualquier modo, el periódico conservador achacaba el triunfo incompleto de los ministeriales, a las fuertes divisiones internas que a la postre no impideron el triunfo de los dos numeros uno de ambos grupos -Rodríguez Batista y González de la Vega-, pero sí de los respectivos dos -Montojo y Galindo-, en beneficio de Genovés. ${ }^{11}$

Las elecciones para la Cámara del Senado a celebrar el dos de septiembre, requería según la Ley, el concurso de representantes de ayuntamientos y mayores contribuyentes para nombrar compromisarios. Sin embargo, la situación desde el mes anterior había sido a nivel de corporaciones locales y provincial, una continuación del inicio del año; ciertamente muchos concejales habían sido suspendidos por un tiempo que superaba ya los cincuenta días de máximo y por su parte, los interinos ya habían superado el cupo de sustitución de éstos. Ello repercutía directamente en el nombramiento de compromisarios para la elección de senadores. Ante la falta de representatividad y falseamiento de la realidad, los conservadores aconsejan la retirada de esta reunión de sus compromisarios, recalcando de nuevo los auténticos intereses liberales:

*Ahora verá el público lo que nosotros vimos desde el primer día, que el único objeto de la suspensión era tener, durante el periodo electoral, diputados de real orden que manejasen a gusto del gobierno la máquina consabida y una comisión provincial amiga que aprobase o desaprobase las actas de la elección municipal, según conviniera a los intereses del partido dominante. Concluida ya las elecciones no hay dificultad en hacer justicia a nuestros amigos de la Diputación..12

Así, el día estipulado para la elección y con una mesa presidida por los representantes Alvarez Jiménez, Francisco Arias, José Bertemati, Esteban Rej de la Cruz, José Calatrigo y Fermín de Urmeneta, fueron elegidos senadores Rios Rosas (noventa votos), Shelly (ochenta y nueve) y Misa (ochenta y nueve).

Esto es a grandes rasgos lo que dio de sî el paquete electoral del primer turno liberal, acaecido en la ciudad de Cádiz en 1881. Como no es dificil observar, nos encontramos con un perfil de funcionamiento muy en la órbita de lo que podremos hallar posteriormente tanto en este ciudad, como en gran parte del espectro español:

(11) Las divisiones internas en el fusionismo se mantuvieron de tal forma que los grupos de González de la Vega y del Toro, desarrollaron vidas paralelas.V. El Comercio, 22, 23 y 24-ag.-y 6-sep.-1881 y El Clamor de Cádiz, 22-ag.-1881.

(12) Dos diputados de los suspendidos por las causas aducidas, Francisco de Paula Regife y el anterior presidente José Ramón de Santa Cruz, hicieron valer en un escrito su derecho al veto de senadores. El gobierno provincial se desentendieron del asunto.V.El Comercio 28-jul.-y 7, 17, 25 y 30.-ag.-1881. 
Uso de la suspensión de cargos por interinos, para asegurar clientelas políticas en las corporaciones y órganos de decisión.

Uso y manipulación de censo, cédulas y demás cauces de identidad.

Uso de personal de la administración pública, para usos de chantaje y manipulación electoral.

Control o intentos de control de cada uno de los cauces constitutivos del hecho electoral: Mesas, compromisarios, recuentos, interventores.

Apoyo y respaldo de los cauces de la administración y gobierno central, para cuyos cauces se vale los poderes intermedios locales y provinciales, aunque a veces los compromisos locales caciquiles o las excesivas divisiones internas, tergiversa mínimamente las previsiones.

Uso y deformación de los órganos de difusión para intentar tapar los amaños consiguientes perjudicando si es posible, el partido de oposición y las formaciones de minoría.

Así, la apertura del censo a partir de 1891 con el Sufragio Universal masculino, vino a ampliar el campo de acción y control del mecanismo, pero en esencia, las pautas ya se estaban marcando desde estas fechas. Un sistema que como el electoral de la restauración venía a consistir en un orden de turno, en donde hasta las críticas y ataques mútuos de un dinámica caciquil, eran recíprocas. En 1881 en Cádiz, son conscientes del sistema político que les y toman una diligente actitud de culpabilidad ajena, y no cejan en atacarse mutuamente de caciques. El propio periodista José María Gómez Colón en un artículo sobre el caciquismo, teorizaba sobre el hecho como fenómeno con resquicios sociológicos y de inevitable absorbencia al colectivo. Sus problemas, sus males, sus sufrimientos al ajeno, sólo tenian un camino, pues ....si hay firmeza para negarse a las pretensiones extralegales, por insignificantesque parezcan, perecerá el caciquismo. Por que será tanto como sacar los dientes al lobo.*13

(13) V. El Clamor de Cádiz, 14-oct.-1881. 